\title{
The Development of a New Sensor for Tool Face Angle Based on Electrical Resistance
}

\author{
Chuan Wu $\mathbb{D}^{1,2}$ Jian Pan $\mathbb{D}^{1,2}$ and Guojun Wen $\mathbb{D}^{1,2}$ \\ ${ }^{1}$ School of Mechanical Engineering and Electronic Information, China University of Geosciences, Wuhan 430074, China \\ ${ }^{2}$ Hubei Intelligent Geological Equipment Engineering Technology Research Center, Wuhan 430074, China \\ Correspondence should be addressed to Guojun Wen; wenguojun@cug.edu.cn
}

Received 15 May 2019; Revised 18 July 2019; Accepted 8 August 2019; Published 15 September 2019

Academic Editor: Everardo Vargas-Rodriguez

Copyright (C) 2019 Chuan Wu et al. This is an open access article distributed under the Creative Commons Attribution License, which permits unrestricted use, distribution, and reproduction in any medium, provided the original work is properly cited.

\begin{abstract}
Trenchless technology is a type of construction that requires pipelines to be laid in a subsurface horizontal hole drilled by a trenchless drilling rig. To prevent drilling accidents during construction, the drilling trajectory needs to be controlled in real time according to the value of the tool face angle. However, existing tool face angle sensors cannot precisely control a trenchless drilling course due to a number of limitations such as low resolution, large temperature drift, and high requirements for accurate installation. In this regard, this paper presented a new tool face angle sensor based on the change in electrical resistance value. The tests mainly include four aspects, namely, accuracy, repeatability, resolution, and temperature drift. Numbers of tests showed that the measurement error was \pm 6 degrees, the repeatability was $2.54 \%$, the minimum resolution was 6 degrees, and the temperature drift had no effect during the temperature changes from 0 to 80 degrees, which proved that the sensor is applicable for practical trenchless working conditions.
\end{abstract}

\section{Introduction}

Trenchless technology is a technique involving drilling a horizontal hole under the surface of the earth and then laying pipelines in the horizontal hole [1]. The equipment used in trenchless technology mainly includes a trenchless drilling rig, a drill bit, and numbers of drill pipes. The drill pipe is clamped by the drilling rig (or rig for short) on the earth's surface and a number of drill pipes can be connected together.

As shown in Figure 1(a), OA is the high side, $O B$ is the normal direction of the drill bit's lip, and $\alpha$ is the high-side tool face angle (called the tool face angle for short). During trenchless construction, when the rig supplies the bit with both forward force and rotary force, the bit is in a balanced state of forces and moves straight ahead (as shown in Figure 1(a)); therefore, the drilling trajectory is not affected by the tool face angle. On the contrary, when the rig supplies the bit with only forward force, the bit is not in a balanced state of force because of the special structure of the bit's lip, which causes an inclination of the drilling trajectory (as shown in Figure 1(b)); therefore, the drilling trajectory is affected by the tool face angle $[2,3]$. Hence, the tool face angle must be measured and adjusted in real time to control the drilling trajectory.

In general, a tool face angle sensor can be classified as conductive, capacitive, photoelectric, or SINS type (strapdown inertial navigation system) according to the working principle [4-7]. However, these sensors cannot precisely control a trenchless drilling course due to a number of limitations such as low resolution, insufficient precision, large temperature drift, overlarge size, and high requirement for accurate installation. Details of these sensors are given in the following sections.

\subsection{Conductive Tool Face Angle Sensor}

1.1.1. Basic Principle. Figure 2 is a sketch map of a conductive tool face angle sensor [4]. VCC stands for the positive pole of the power while GND stands for the negative pole of the power. The sensor consists of two coaxial cyclic structures, and each of the cyclic structures is pasted with a conductive 


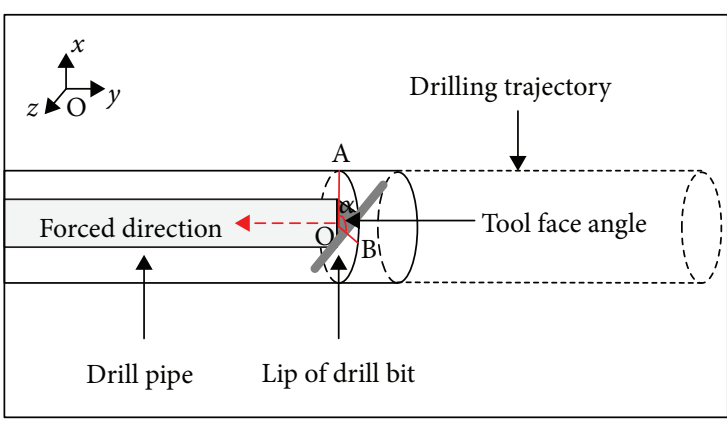

(a)

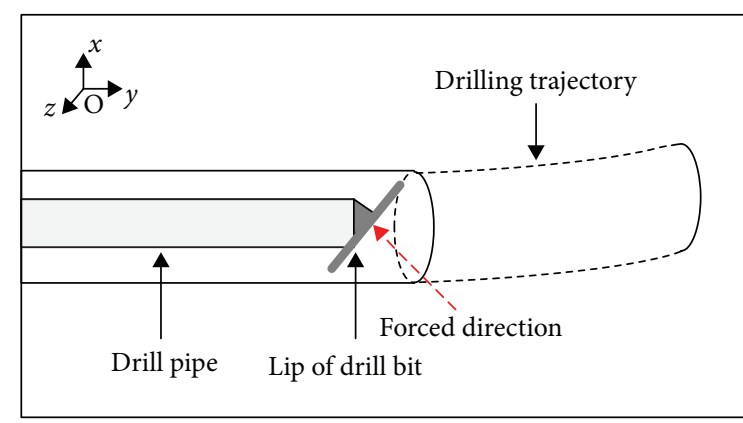

(b)

FIGURE 1: Sketch map of the controlling drilling trajectory by tool face angle. (a) Sketch map of the trenchless drilling construction when the drilling trajectory is straight. (b) Sketch map of the trenchless drilling construction when the drilling trajectory is in inclination.

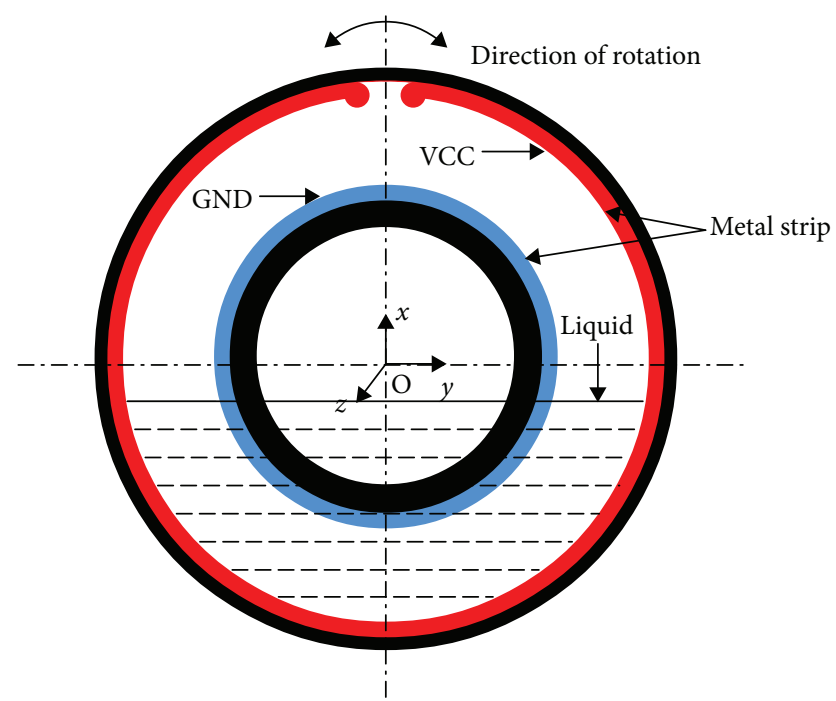

Figure 2: Sketch map of a conductive tool face angle sensor.

metal strip. One of the metal strips is a resistive layer. The metal strip on the inner cyclic structure is continuous and is connected to the GND while the metal strip on the outer cyclic structure is not continuous and is connected to the VCC. Inside the sensor, there is an amount of conductive liquid that is always in the lower part of the chamber and the liquid connects the metal strips of the VCC and GND. When the tool face angle changes (i.e., when the sensor rotates), the connecting position of the metal strips changes accordingly, namely, the connecting length of the metal strips between the VCC and GND changes. In other words, the total electrical resistance of the circuit becomes different. Therefore, the tool face angle can be measured founded on the change of the electrical resistance.

1.1.2. Inadequacies. The conductivity of the liquid is greatly affected by temperature, which will cause a large temperature drift in the sensor and, therefore, results in a large measurement error. Furthermore, the splashing and adhesion of the liquid can easily lead to misinterpretation of the sensor. In brief, this sensor has a big measurement error and is too sensitive to temperature.

\subsection{Capacitive Tool Face Angle Sensor}

1.2.1. Basic Principle. A sketch map of a capacitive tool face angle sensor is shown in Figure 3 [5]. VCC is the positive power supply while the GND is the negative power supply. As shown in Figure 3(a), the structure of the sensor is a sealed cylindrical chamber that is pasted with electrodes on both sides. One side of the chamber is pasted with a number of electrodes which are all connected to the VCC (in general, the number is 16), while the other side is completely covered with one electrode connected to the GND. There is an amount of liquid which is always in the lower part of the chamber. Therefore, a number of capacitors are formed between the positive and the negative electrodes. The position of each capacitor can be numbered. When the tool face angle changes, the capacitor values will change accordingly. Therefore, the tool face angle can be measured based on the change of the numbered capacitors.

1.2.2. Inadequacies. The medium of the capacitor in the sensor is liquid, so the dielectric constant of the liquid is greatly affected by temperature, which will cause a large temperature drift in the sensor and, therefore, results in a large measurement error. Meanwhile, the adhesion of the liquid can cause interference among the capacitors, which will lead to misinterpretation of the sensor and result in a large measurement error. Furthermore, capacitors on the same sensor can easily interfere with each other when there are larger numbers of capacitors. In most cases, the number of the capacitors is small (generally, it is 16, so the minimum resolution is 360 / $16=22.5$ degrees). As the resolution of the sensor depends directly on the number of capacitors, the resolution of the sensor is generally low.

\subsection{Photoelectric Tool Face Angle Sensor}

1.3.1. Basic Principle. Figure 4 is a sketch map of a photoelectric tool face angle sensor [6]. The structure of the sensor is a sealed cylindrical chamber that is installed with luminescent tubes on one side (generally installed with 12 luminous diodes) and receiving tubes on the other. The light signals sent from the luminescent tubes can be received by the receiving tubes. There is a ball that rolls freely in the chamber between the luminescent tubes and the receiving tubes, so it is always at the bottom of the chamber. The position of each 


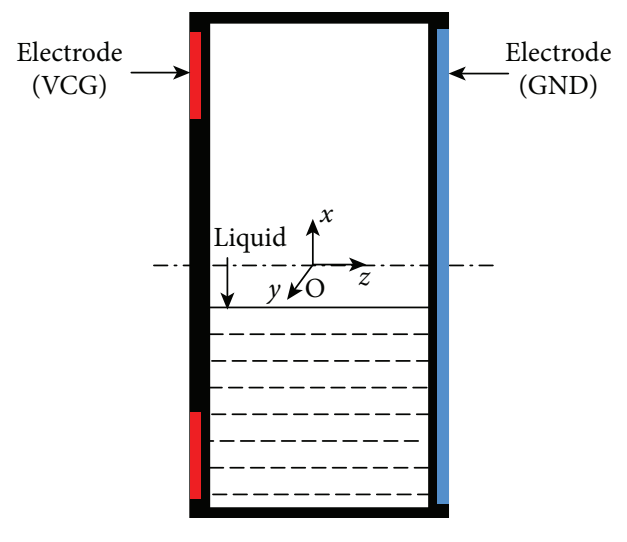

(a)

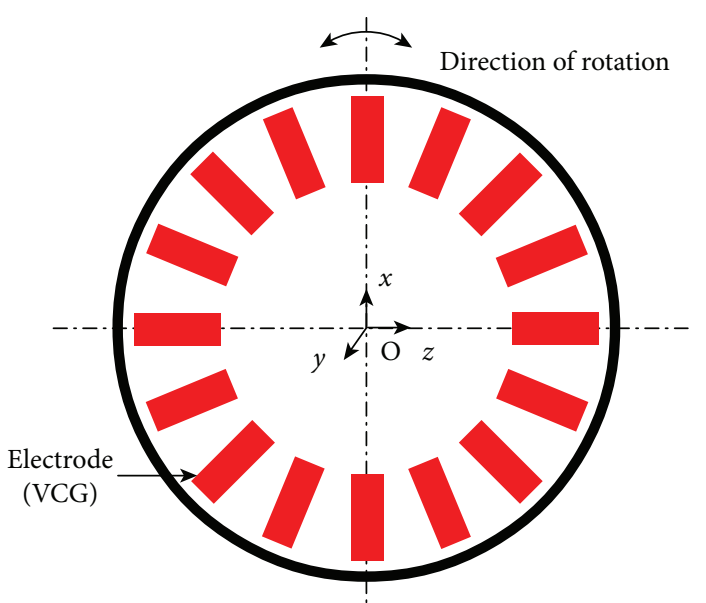

(b)

FIGURE 3: Sketch map of a capacitive tool face angle sensor. (a) The overall structure of the sensor. (b) The circumferential distribution of the electrodes.

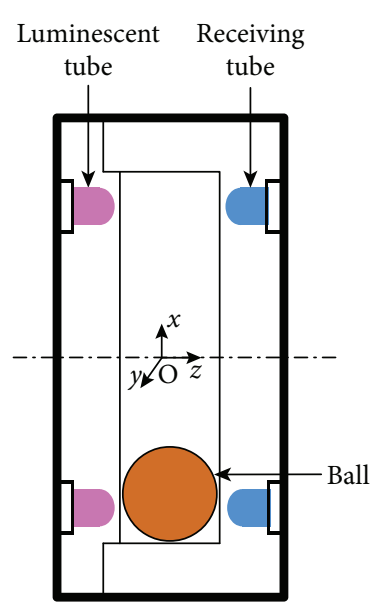

(a)

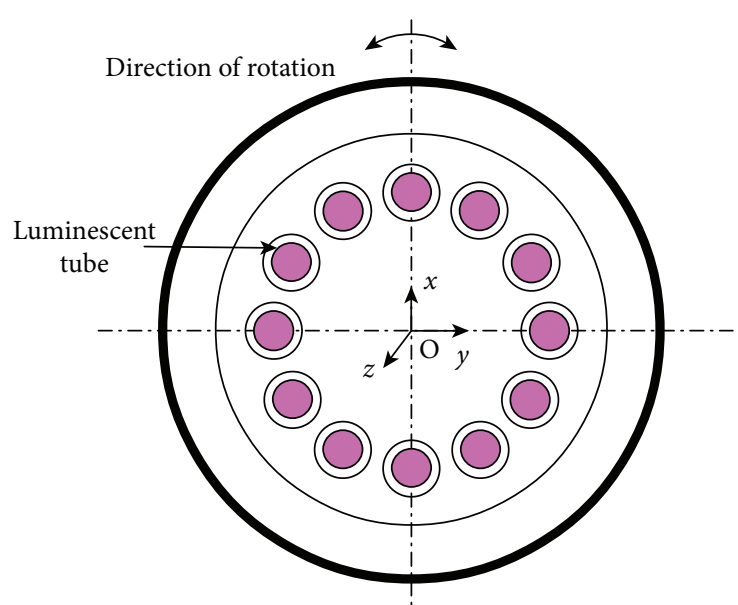

(b)

FIGURE 4: Sketch map of a photoelectric tool face angle sensor. (a) The structure of the sensor. (b) The circumferential distribution of the luminescent tubes.

receiving tube can be numbered. When the tool face angle changes, the light signal sent by the lowest luminescent tube is blocked by the ball so that the value of the receiving tube changes accordingly. Therefore, the tool face angle can be measured based on the signals outputted by the numbered receiving tube.

1.3.2. Inadequacies. The resolution of the sensor depends on the number of luminescent tubes or receiving tubes. However, as trenchless construction has limitations on size, the number of luminescent tubes is generally small (in most cases, it is 12 or, in a few cases, 24 , so the minimum resolution is $360 / 24$ $=15$ degrees). As a result, the resolution is low and the measurement is not accurate because of the resolution.

\subsection{Measurement of Tool Face Angle Based on SINS}

1.4.1. Basic Principle. Strapdown inertial navigation system (SINS) consists of a triaxial accelerometer and triaxial gyroscope (or triaxial electromagnetic gauge). By using the output data of the triaxial accelerometer and the triaxial gyroscope, any space attitude of an object (including the tool face angle, velocity, acceleration, and angular velocity) can be determined through complex mathematical modeling and calculation [7].

1.4.2. Inadequacies. Strictly speaking, SINS is a measurement system composed of a plurality of sensors arranged in space. By now, the triaxial electromagnetic gauge of SINS has been replaced by a triaxial gyroscope as it is extremely susceptible to electromagnetic interference. Although SINS is accurate and precise, it is not suitable for trenchless constructions for the following reasons:

(1) A fiber-optic gyroscope is generally used as the triaxial gyroscope and its measurement accuracy is positively related to its size. To ensure accurate measurements, the size of the triaxial gyroscope is generally large, which is also contradictory to the small spatial size near the trenchless drilling bit 


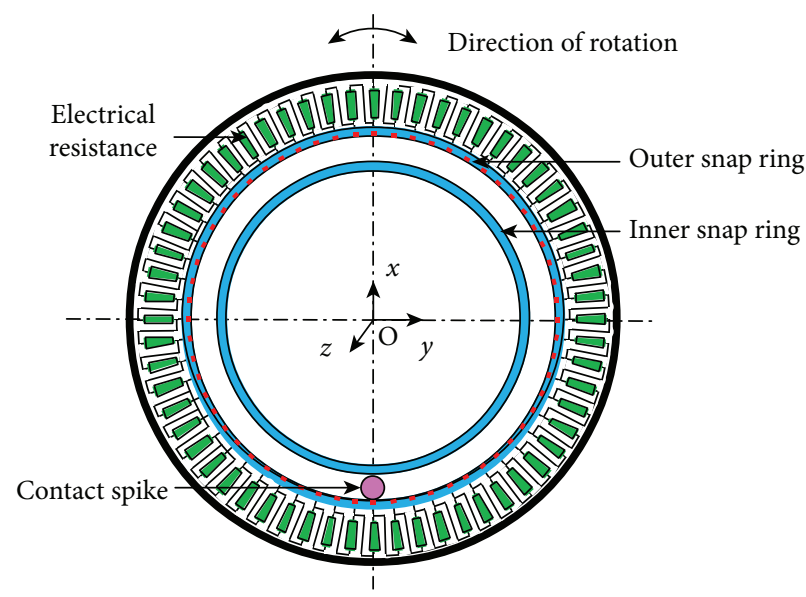

(a)

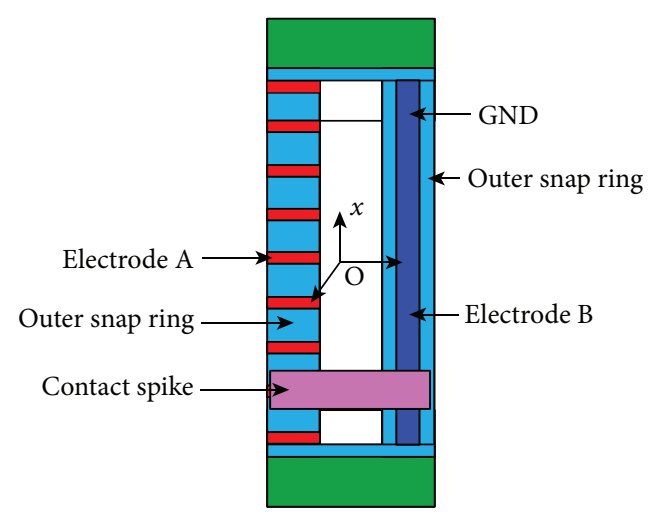

(b)

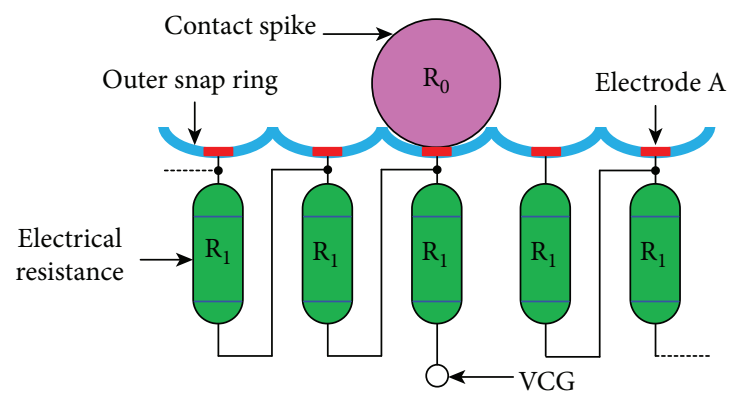

(c)

Figure 5: Structure of the sensor. (a) Basic components, (b) connection of the electrodes, and (c) detailed connection of the electrical resistances.

(2) To calculate the tool face angle, triaxial accelerometers and triaxial gyroscopes must be spatially arranged in a certain pattern. Therefore, the sensor must be installed accurately and precisely, which is difficult to realize in trenchless constructions

(3) The triaxial gyroscope (fiber-optic gyroscope) is a precise optical sensor that has high requirements for vibration electrical resistance. However, the working conditions during trenchless construction are extremely complex and harsh where the requirements for vibration electrical resistance cannot be guaranteed

(4) Temperature drift in a triaxial gyroscope is large and is still an unsolved problem, though a number of researchers have carried out studies of algorithms to treat temperature drift [8-10]

(5) Due to the Faraday magneto-optic effect, the polarization plane of the light in the triaxial gyroscope will rotate [11]. As a result, the gyroscope can still be affected by electromagnetic interference. In most trenchless constructions, however, there is extrahigh-voltage cables that can generate great electromagnetic interference

1.5. Analysis of Existing Sensors. In conclusion, existing sensors cannot precisely control a trenchless drilling course due to a number of limitations such as low resolution, insufficient precision, large temperature drift, overlarge size, and high requirement for accurate installation. To be specific, for the conductive tool face angle sensor, the measurement error is large due to the large temperature drift and the splashing and adhesion of the liquid. For the capacitive tool face angle sensor, the measurement error is also large because of the large temperature drift and the splashing and adhesion of the liquid. Additionally, the resolution of the sensor is too low (only $22.5^{\circ}$ ). For the photoelectric tool face angle sensor, the resolution is too low (only $15^{\circ}$ ). Strictly speaking, SINS is not a sensor. It is not suitable for trenchless constructions because of a number of limitations such as overlarge size, high requirements for both accurate installation and working conditions, and temperature drift. In this regard, a new tool face angle sensor was designed in this article on the basis of practical working conditions. The sensor could measure the tool face angle through the change of electrical resistance values. Compared to existing sensors (excluding the SINS-based sensor), this sensor is more suitable for trenchless constructions as it has a higher resolution and sufficient precision as well as effectively solving the problem of temperature drift.

\section{Basic Principle of the Designed Sensor}

2.1. Basic Principle. Figure 5 is the sketch map of the designed sensor's structure. VCC is connected to the positive pole of 


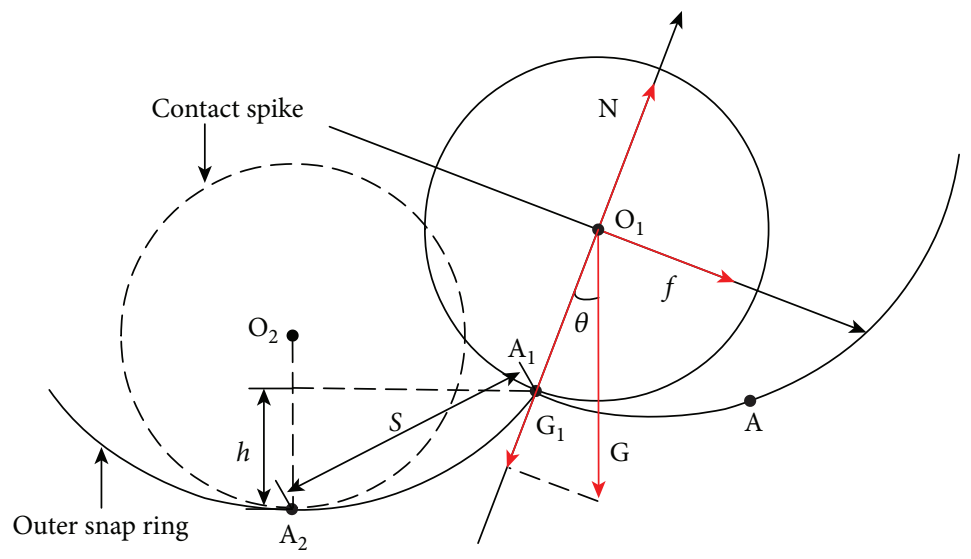

Figure 6: Forces acting on the contact spike when the tool face angle changes.

the power supply while GND is connected to the negative pole of the power supply. As shown in Figure 5(a), a circular channel is formed by the inner and the outer snap rings where there is a cylindrical metal contact spike that could roll freely in the channel. As shown in Figure 5(b), there are 60 grooves in the channel and electrode $\mathrm{A}$ is pasted on one side of the groove and electrode $\mathrm{B}$ on the other side. As shown in Figure 5(c), below each electrode A, there is an electrical resistance $R_{1}$ and a string is formed by all of the electrical resistances $R_{1}$ that are connected in series. The ends of the string of the electrical resistance are not connected together and one end of the string is connected to the VCC. Electrode B is connected to the GND. Given that the current flows starting from the VCC and pass through the resistances string,the electrode $\mathrm{A}$, the contact spike, the electrode $\mathrm{B}$, and then connected to the GND, thereby forming a current loop.

The inner surface of the outer snap ring is arc shaped (as shown in Figure 5(c)), so that when the tool face angle changes, the contact spike will fall along the channel to the lowest point on the arc-shaped surface under the force of gravity. As a result, the number of the electrical resistances in the circuit will also change. That is to say, the total electrical resistance value in the circuit will change. Therefore, the tool face angle could be measured according to the mathematical relation between the total electrical resistance value and the tool face angle.

2.2. Key Performance Indexes. From the working principle of the sensor, we can see that the value of the measured tool face angle is positively related to the total electrical resistance value in the circuit, then the tool face angle can be expressed as follows:

$$
\begin{aligned}
\phi & =6 \frac{R-R_{0}-R_{1}}{R_{1}} \\
& =6 \frac{R-R_{0}\left[1+\alpha_{R_{0}}\left|t_{2}-t_{1}\right|\right]-R_{1}\left[1+\alpha_{R_{1}}\left|t_{2}-t_{1}\right|\right]}{R_{1}\left[1+\alpha_{R_{1}}\left|t_{2}-t_{1}\right|\right]},
\end{aligned}
$$

where $\phi$ is the tool face angle; $R$ is the total electrical resistance value between the VCC and GND; $R_{0}$ is the electrical resistance value of the contact spike; $R_{1}$ is the value of a single electrical resistance below electrode $\mathrm{A} ; \alpha_{R_{0}}$ is the temperature coefficient of electrical resistance $R_{0} ; \alpha_{R_{1}}$ is the temperature coefficient of electrical resistance $R_{1}$; and $t_{2}-t_{1}$ is the working time of the sensor.

In expression ((1)), the value of $R$ can be measured in real time through the hardware circuit; $R_{0}, R_{1}, \alpha_{R_{0}}$, and $\alpha_{R_{1}}$ are constants that need to be determined for designing the sensor.

In the actual measurement circuit, the value of $R$ is obtained by measuring the current of the circuit, so expression $((1))$ can also been expressed as follows:

$$
\phi=6 \frac{R-R_{0}-R_{1}}{R_{1}}=6 \frac{E-I R_{0}-I R_{1}}{I R_{1}},
$$

where $E$ is the supply voltage of the circuit and $I$ is the measured current of the circuit.

2.2.1. Minimum Resolution. For the designed sensor, there are 60 electrical resistances in total that are uniformly pasted on the outer snap ring, so the sensor's minimum resolution can be calculated directly by

$$
A_{R}=\frac{360}{n}=\frac{360}{60}=6^{\circ}
$$

where $A_{R}$ is the minimum resolution of the sensor and $n$ is the number of electrical resistance $\left(R_{1}\right)$ between the VCC and GND.

2.2.2. Key Dimension Parameters. As shown in Figure 6, in ideal conditions, if the tool face angle changes at the rate of the minimum resolution, then the contact spike moves on the outer snap ring from point $\mathrm{A}$ to point $\mathrm{A}_{2}$. During this process, the contact spike decelerates to point $A_{1}$ under the action of gravitational potential energy. So, when the velocity of the contact spike at point $A_{1}$ is greater than zero, the sensor can achieve the desired function. 
TABLE 1: Expressions for resolving the forces of Figure 6.

\begin{tabular}{lcc}
\hline Expressions & Expression no. & Explanation \\
\hline$E_{G}=m g h$ & (i) & Gravitational potential energy \\
$W_{f}=f s$ & (ii) & Friction work \\
$E_{v}=1 / 2 m v^{2}$ & (iii) & Kinetic energy \\
$N=G_{1}$ & (iv) & Supporting force \\
$G_{1}=G \cos \theta$ & (v) & Component force of gravity \\
$f=N \mu$ & (vi) & Friction \\
\hline
\end{tabular}

Now, we analyze the forces on the contact spike at point $A_{1}$ based on the energy conservation law illustrated in

$$
E_{G}=W_{f}+E_{v},
$$

where $E_{G}$ is the gravitational potential energy of the contact spike, $W_{f}$ is the work done by friction, and $E_{v}$ is the kinetic energy of the contact spike at point $A_{1}$.

The expressions as shown in Table 1 can be obtained by resolving the forces in Figure 6.

$m$ is the mass of the contact spike; $g$ is the gravitational constant; $f$ is the friction; $v$ is the velocity of the contact spike at $\mathrm{A}_{1} ; G$ is the gravity; $G_{1}$ is the component force of $G ; N$ is the supporting force; and $\mu$ is the friction coefficient.

From expressions (4) through (vi) we have

$$
v=\sqrt{2 g(h-\mu s \cdot \cos \theta)}>0 \Longrightarrow h>\mu s,
$$

where $v$ is the velocity of the contact spike at $\mathrm{A}_{1}$.

In this design, $h=0.43 \mathrm{~mm}, s=2.53 \mathrm{~mm}$, and $\mu=0.08$ (the contact spike is made of copper; the outer snap ring is made of polytetrafluoroethylene and is also lubricated. The data were measured by CF-02 produced by Chen Rui Intelligent Equipment Co., Ltd.).

2.2.3. Key Circuit Parameters. As can be known from expression (1), parameters for designing the circuit are mainly $R_{0}, R_{1}, \alpha_{R_{0}}$, and $\alpha_{R_{1}}$. Here, we further discuss and analyze these parameters.

The contact spike is equivalently a conductor and its temperature drift should be as small as possible. So, we select the copper to make the contact spike and the measured value of electrical resistance $R_{0}$ was about $0.03 \Omega$.

To reduce the temperature drift of $R_{1}, R_{1}$ should be a precise electrical resistance with a small temperature coefficient. In this article, we select $R_{1}=100 \Omega$ (VTA56, VPG Co., Ltd).

The operating temperature range of the designed sensor was $-20^{\circ} \mathrm{C}$ to $80^{\circ} \mathrm{C}$. In this temperature range, the maximum variation of $R_{0}$ is $0.0118 \Omega$ and $R_{1}$ is $0.002 \Omega$. So, the temperature will not influence the accuracy of the designed sensor.

\section{Tests}

3.1. Test Equipment. A standard angular measuring platform and a water bath were used to test the measurement error and temperature drift of the sensor. The standard angular mea- suring platform could realize the rotation of the tool face angle within the range of 0-360 degrees with the resolution of 1 degree. The water bath could precisely control the temperature of the water within $0-100^{\circ} \mathrm{C}$ with the resolution of $1^{\circ} \mathrm{C}$.

3.2. Test Procedure. The sensor was fixed to the angular measuring platform and then put into the water bath after being sealed. By adjusting the tool face angle of the platform and the temperature of the water bath, the tests were divided into two groups. One was in the same temperature, by adjusting the tool face angle $\left(0-360^{\circ}\right)$ of the platform to test the measuring error (called test 1 for short), the other was in the same tool face angle, by adjusting the temperature (from room temperature to $80^{\circ} \mathrm{C}$ ) of the water bath to test the temperature draft (called test 2 for short). In the above two kinds of tests, each test time was 1 hour and the times of tests was 20 times repeatedly.

3.3. Test Results. The data outputted from the sensor were named as the "measured value" and the data read from the platform were named as "standard value." Figure 7(a) illustrates a photograph of the sensor. Figure $7(\mathrm{~b})$ illustrates one of the test results of "test 1" which contained the maximum error and Figure 7(c) illustrates one of the test results of "test 1" which contained the maximum repeatability. In Figures 7(b) and 7(c), the abscissa is the standard tool face angle $\left({ }^{\circ}\right)$ while the ordinate is the measured tool face angle $\left({ }^{\circ}\right) . E_{\max }$ is the maximum measurement error and $\sigma_{\max }$ is the maximum deviation. Figure $7(\mathrm{~d})$ illustrates one of the test results of "test 2" which contained the maximum temperature draft (the tool face angle is $150^{\circ}$ ). The abscissa is the test temperature $\left({ }^{\circ} \mathrm{C}\right)$ while the ordinate is the tool face angle $\left({ }^{\circ}\right)$.

It can be concluded from Figure 7 that

(1) From Figure 7(b), we can obtain the following conclusions. (a) The maximum error of the sensor is \pm $6^{\circ}\left(E_{\max }=6\right)$, the curve of the measured values as "step-shaped," and most of the measured values are smaller than the standard values. The reason is that the minimum resolution of the sensor was $6^{\circ}$. When the tool face angle is changed a little (less than $6^{\circ}$ ), the output of the sensor remained the same due to the force of the sensor groove structure, thereby causing that the measured value is smaller than the standard value. (b) Some measured values are larger than the standard values. The reason is that the minimum resolution of the sensor is $6^{\circ}$. When the tool face angle is changed greatly (greater than $6^{\circ}$ ), the output of the sensor increased by $6^{\circ}$, thereby causing that the measured value is greater than or equal to the standard value. In addition, the structure of the sensor can also cause it. The reason is that there is a rolling contact in the structure of the sensor. So when the contact is poor, the resistance value of the measuring circuit is slightly increased. Because the resistance value is proportional to the tool face angle value, the measured values are slightly larger than the 


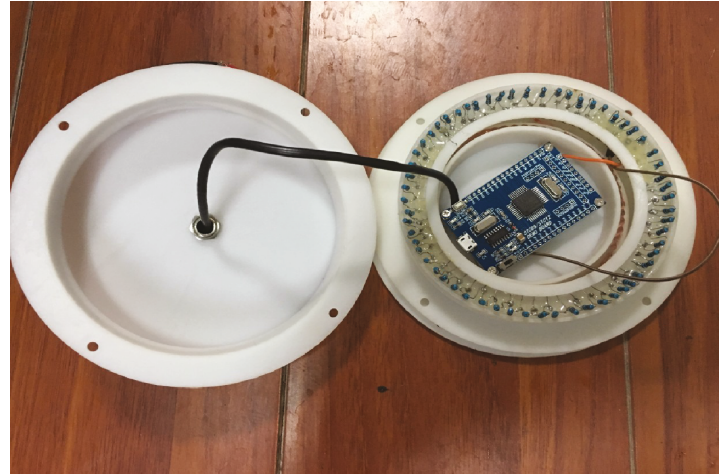

(a)

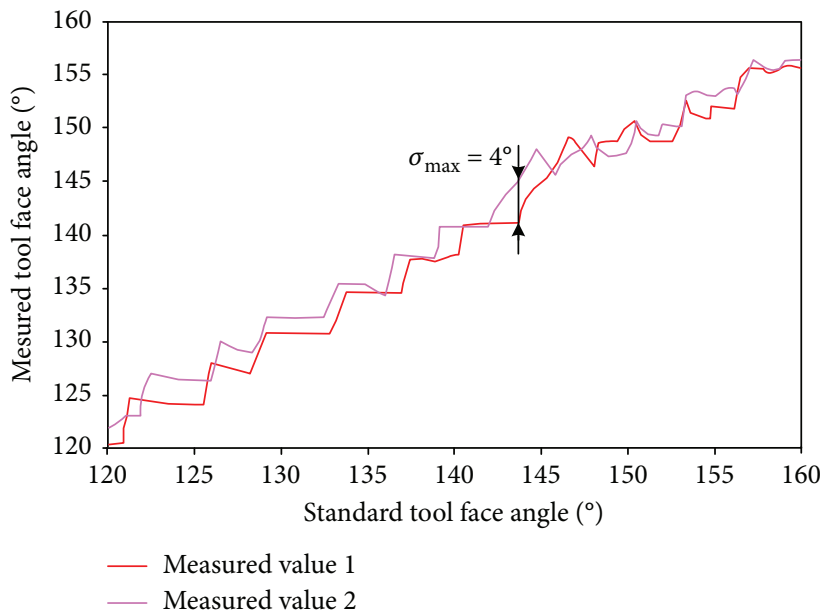

(c)

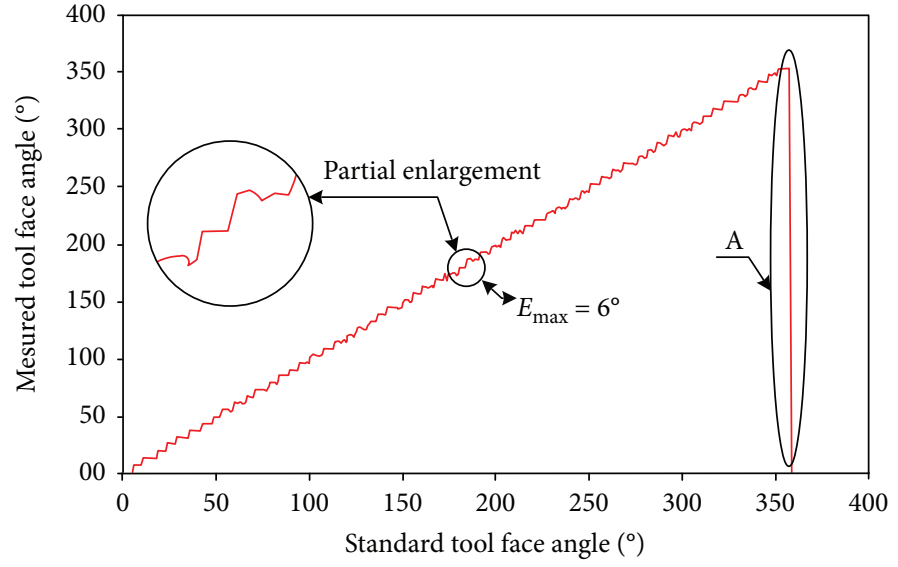

(b)

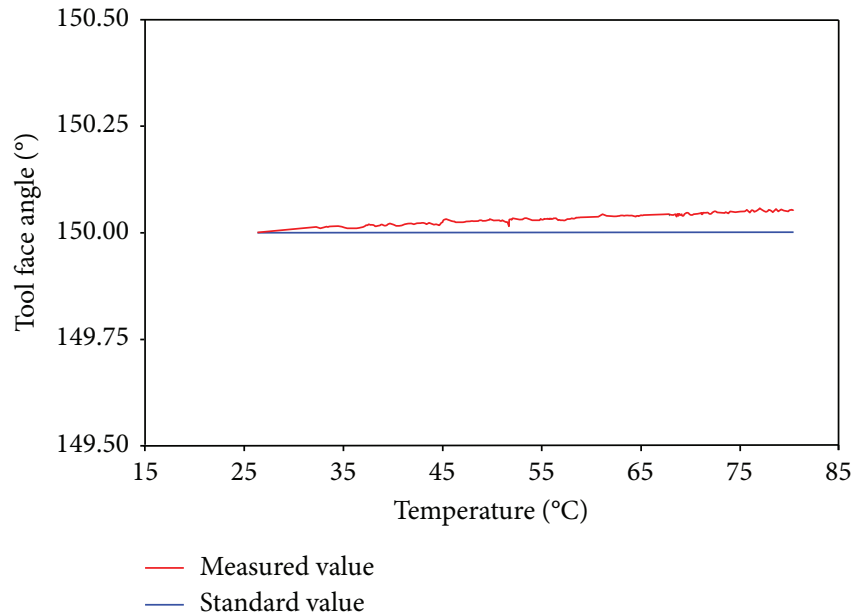

(d)

Figure 7: (a) The sensor photograph. (b) The test results contained the maximum error. (c) The test results contained the maximum deviation. (d) The test results contained the maximum temperature draft.

standard values. (c) Point A showed that the position when the tool face angle is $0^{\circ}$ is the same as the position when the tool face angle is $360^{\circ}$. This is why the measured value is $0^{\circ}$, but the corresponding standard value is $360^{\circ}$

(2) From Figures 7(c) and 7(d), we can obtain the following conclusions. As shown in Figure 7(c), the maximum deviation of the sensor is $4^{\circ}\left(\sigma_{\max }=4\right)$. By substituting the average deviation (the average deviation is 3.05) into the sensor repeatability formula, we can obtain that the repeatability of the sensor is 2.54\%. As shown in Figure 7(d), the output of the sensor will increase with the temperature increasing, and the measured values are greater than the standard values. The reason is the resistance of the sensor increased with the temperature increased, which lead to a proportional increase in the output value of the sensor. But the effect of temperature on the sensor's measurement accuracy can be neglected. In other words, there is almost no temperature drift in the sensor
(3) It can be known from expressions (1) and (2) that the measured tool face angel is proportional to the measured electrical resistance. Therefore, the ordinate of Figure 7 (b) can also be converted into the measured electrical resistance when multiplied by the corresponding coefficient, that is, the shape of the curve shown in Figure 7(b) can also represent the relationship (in which the abscissa is the standard tool face angle and the ordinate is the measured electrical resistance)

\section{Conclusions}

(1) Aiming to better adapt to the trenchless construction environment, this article presentes a new tool face angle sensor based on the change of the electrical resistance value. Relevant tests shown that the minimum resolution of the sensor is $6^{\circ}$, which is much better than that of existing sensors; the measurement error of the sensor is $\pm 6^{\circ}$, which is mainly caused by the resolution of the sensor; and the repeatability is $2.54 \%$. (2) Numbers of tests have shown that the electrical 
resistance value increased as the temperature increased, and which results in a slight increase in the sensor output. But thanks to the use of precision temperature-resistant drift resistors, this increase has little effect on the measurement accuracy and is almost negligible. That is to say, no temperature drift is nearly in the sensor. (3) The structural design and the rolling conduction method of the sensor result in the measurement curve similar to a "step-shaped" rather than a complete line, which will lead to measurement errors. In further research, the way to further reduce the measurement error is to change the structure design and the conduction method of the sensor.

\section{Data Availability}

No data were used to support this study.

\section{Conflicts of Interest}

The authors declare that they have no conflicts of interest.

\section{Acknowledgments}

This work was supported by the Natural Science Foundation of China (61733016), the Nature Science Foundation of Hubei Province (2018CFB349), the Hubei Provincial Technical Innovation Major Project (2018AAA035), and the Fundamental Research Funds for the Central Universities, China University of Geosciences (Wuhan) (CUG170655).

\section{References}

[1] C. Wu, G. Wen, X. Wu et al., "Calculation method of the pull-back force for cable laying of the trenchless completed power pipeline," Journal of Coastal Research, vol. 73, pp. 681686, 2015.

[2] M. Najafi, Trenchless technology: planning, equipment, and methods, McGraw-Hill Education, New York, NY, USA, 2013.

[3] C. Wu, G. J. W, X. M. Wu et al., "New algorithm for tool face angle in measurement while drilling system," International Journal of Earth Sciences and Engineering, vol. 4, pp. 428436, 2016.

[4] G. Baker, A. W. Chau, and J. E. Mercer, "Technique for steering and monitoring the orientation of a powered underground boring device," 1987, U.S. Patent 4,714,118,.

[5] A. J. Rider, "Angle sensor for a steerable boring tool," 1992, US Patent 5,174,033.

[6] Y. L. Hu, X. M. Wu, and Y. H. Wang, "Development of newtype tool face angle sensor," Journal of Transducer Technology, vol. 24, no. 1, pp. 50-52, 2005.

[7] J. Zhang, J. Li, X. Zhang, X. Che, Y. Huang, and K. Feng, "Analysis and compensation of modulation angular rate error based on missile-borne rotation semi-strapdown inertial navigation system," Sensors, vol. 18, no. 5, p. 1430, 2018.

[8] X. Chen and C. Shen, "Study on temperature error processing technique for fiber optic gyroscope," Optik, vol. 124, no. 9, pp. 784-792, 2013.

[9] A. M. Kurbatov and R. A. Kurbatov, "Temperature characteristics of fiber-optic gyroscope sensing coils," Journal of Communications Technology and Electronics, vol. 58, no. 7, pp. 745-752, 2013.
[10] M. Narasimhappa, J. Nayak, M. H. Terra, and S. L. Sabat, "ARMA model based adaptive unscented fading Kalman filter for reducing drift of fiber optic gyroscope," Sensors and Actuators A: Physical, vol. 251, pp. 42-51, 2016.

[11] X. Li, P. Liu, X. Guang, Z. Xu, L. Guan, and G. Li, “Temperature dependence of faraday effect-induced bias error in a fiber optic gyroscope," Sensors, vol. 17, no. 9, p. 2046, 2017. 


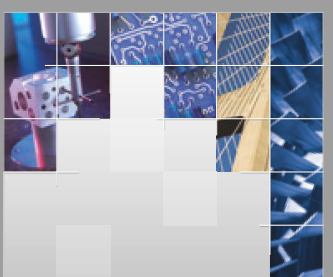

\section{Enfincering}
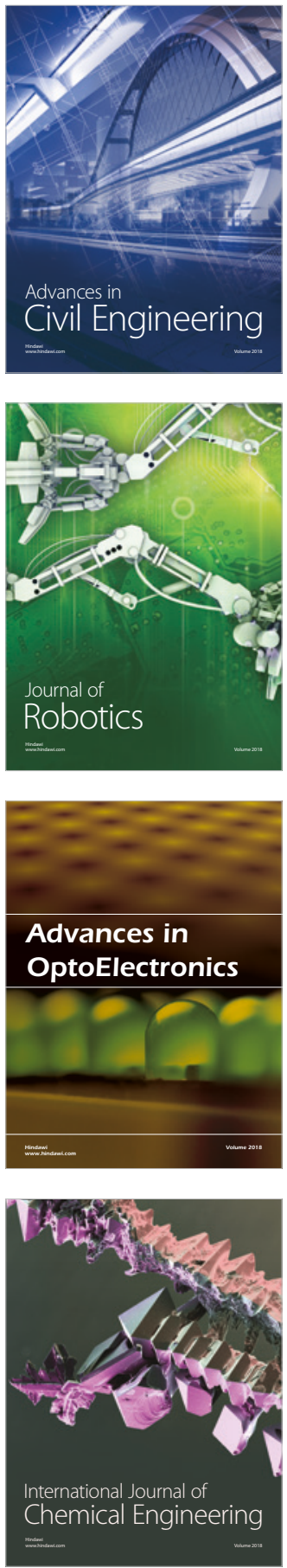

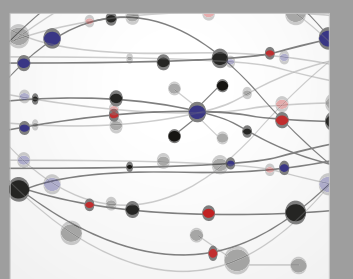

\section{Rotating \\ Machinery}

The Scientific World Journal

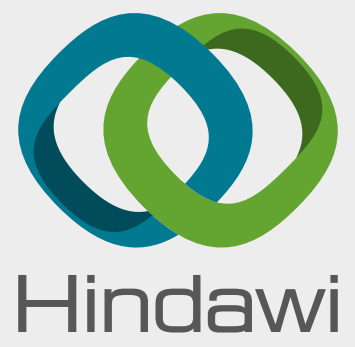

Submit your manuscripts at

www.hindawi.com
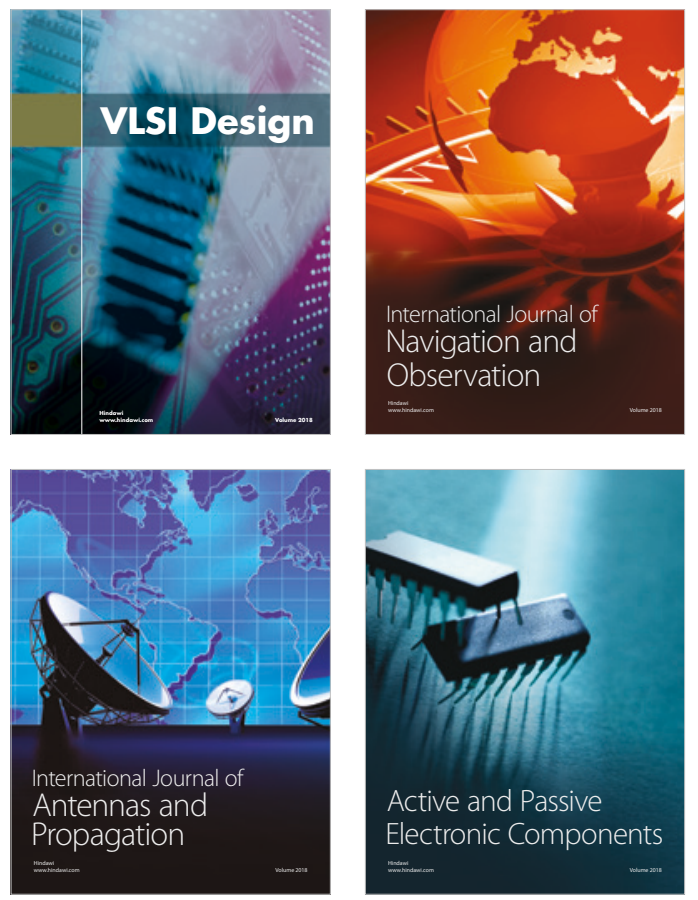
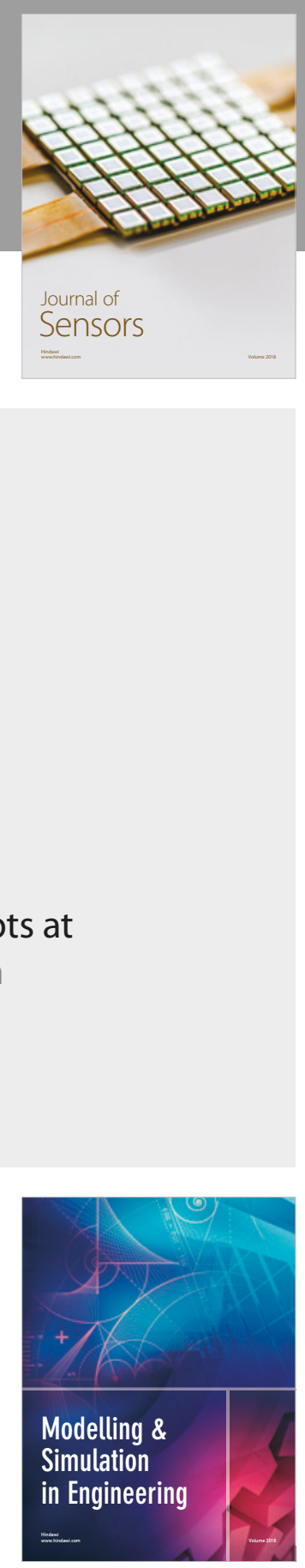

\section{Advances \\ Multimedia}
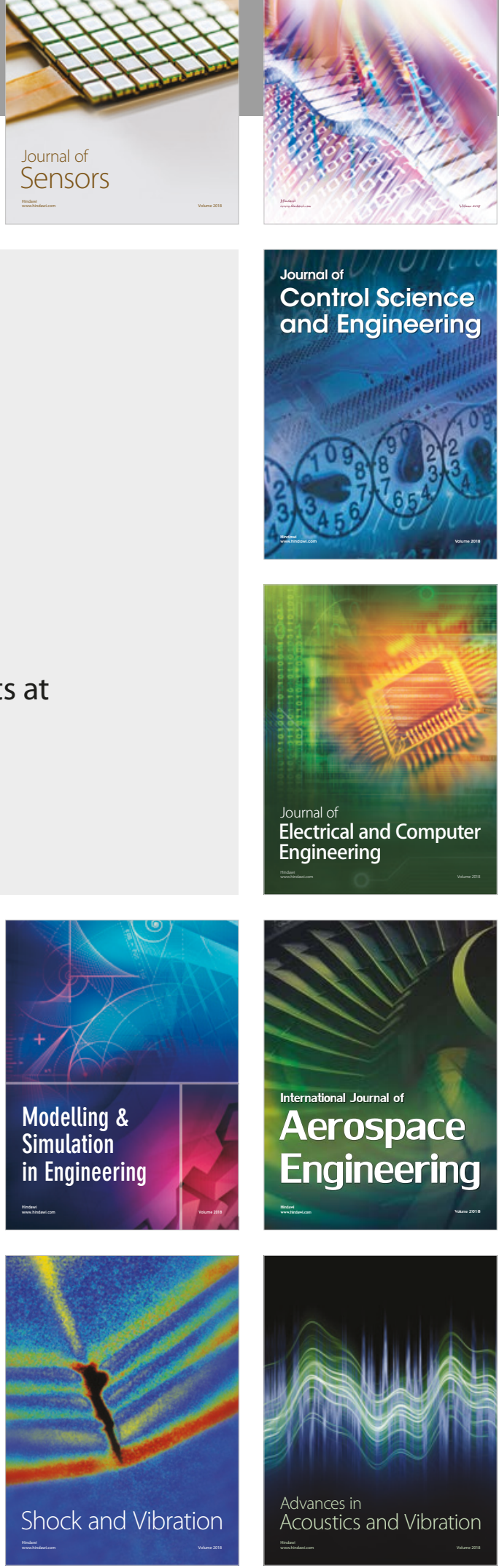